\title{
Investigation of the stimulation capabilities of a high-resolution neurorecording probe for the application of closed-loop deep brain stimulation*
}

\author{
Thomas Tarnaud, Emmeric Tanghe, Sebastian Haesler, Carolina Mora Lopez, \\ Luc Martens, Wout Joseph
}

\begin{abstract}
Deep brain stimulation is an established surgical treatment for several neurological and movement disorders, such as Parkinson's disease, in which electrostimulation is applied to targeted deep nuclei in the basal ganglia through implanted electrode leads. Recent technological improvements in the field have focused on the theoretical advantage of current steering and adaptive (closed-loop) deep brain stimulation. Current steering between several active electrodes would allow for improved accuracy when targeting the desired brain structures. This has the additional benefit of avoiding undesired stimulation of neural tracts that are related to side effects, e.g. internal capsule fibres of passage in subthalamic nucleus deep brain stimulation. Closed-loop deep brain stimulation is based on the premise of continuous recording of a proxy for pathological neural activity (such as beta-band power of measured local field potentials in patients with Parkinson's disease) and accordingly adapting the used stimulus parameters. In this study, we investigate the suitability of an existing highresolution neurorecording probe for high-precision neurostimulation. If a subset of the probe's recording electrodes can be used for stimulation, then the probe would be a suitable candidate for closed-loop deep brain stimulation. A finiteelement model is used to calculate the electric potential, induced by current injection through the high-resolution probe, for different sets of active electrodes. Volumes of activated tissue are calculated and a comparison is made between the highresolution probe and a conventional stimulation lead. We investigate the capability of the probe to shift the volume of activated tissue by steering currents to different sets of active electrodes. Finally, safety limits for the injected current are used to determine the size of the volume in which neurons can be activated with the relatively small electrodes patches on the highresolution probe.
\end{abstract}

\section{INTRODUCTION}

Deep brain stimulation is a surgical treatment for movement and neurological disorders, such as Parkinson's disease [1] or essential tremor [2], in which a current is applied through electrodes that are placed in deep brain nuclei, such as the subthalamic nucleus (STN), the globus pallidus (GP) or the ventral intermediate nucleus (Vim) of the thalamus. In practice, suboptimal placement of the electrode leads or high current injections can result in stimulation-related side-effects, such as facial contractions, ocular deviations, dysarthria, mood and cognitive changes, due to current spread outside the target

*Research supported by FWO-project G046816N. T. Tarnaud is a PhD Fellow of the FWO-V (SB) (Research Foundation Flanders, Belgium).

E. Tanghe is a Post-Doctoral Fellow of the FWO-V (Research Foundation Flanders, Belgium).

T. Tarnaud, W. Joseph, L. Martens, and E. Tanghe are with the Department of Information Technology, Ghent University/IMEC, region [3]. Consequently, non-uniform volumes of tissue activated (VTA), would allow targeting with high precision a nucleus or fibre pathway. This improved stimulation accuracy would result in a reduction of undesired stimulation-induced side effects and a decrease of the total injected charge. Furthermore, studies have shown a topographical organization of the neuronal projections to and from the STN $[4,5,6,7,8]$, even indicating a somatotopic mapping of motor functions in humans. It has been theorized that access to this topology of the subthalamic nucleus, through high-resolution targeting of the VTA, could result in better treatment of movement disorders with deep brain stimulation [9]. In conclusion, localized micro-stimulation by a high-resolution array could be beneficial, both to elucidate the therapeutic mechanism of deep brain stimulation by simultaneously stimulating and recording the evoked response, and as a novel DBS technology for treatment [9]. The high number of active electrodes could be controlled, through a closed-loop implementation of (adaptive) deep brain stimulation [10]. To this end, a proxy for neurological pathology can be used, such as beta-band power in the spectrum of the local field potentials measured by the recording electrodes.

The goal of this study is to explore the potential of an existing high-resolution recording probe [11], for closed-loop deep brain stimulation and high-precision targeting of neuron subpopulations. Due to the small electrode areas, relatively high charge densities are necessary to induce a reasonable size of the VTA. Consequently, safety limits as reported in literature for micro-electrodes are used, to determine the upper bound of current that can be injected $[12,13,14]$.

\section{METHODS}

An existing high-resolution recording probe [11] and a conventional deep brain stimulation lead (Mo. 3389, Medtronic Inc., Minneapolis, MN, USA) are modeled in Sim4life (Sim4life, ZMT Zurich MedTech AG), see Fig. 1. The recording probe consists of 960 rectangular pixels, each containing a single $(12 \mu \mathrm{m})^{2}$ electrode patch. A finiteelement method is used to solve the Ohmic electroquasi-static equation:

$$
\nabla \cdot \sigma \nabla \phi=0
$$

Technologiepark 15, 9052 Zwijnaarde, Belgium. (e-mail: thomas.tarnaud@ugent.be).

S. Haesler is with Neuroelectronics Research Flanders, IMEC, Kapeldreef 75, 3001 Leuven, Belgium.

C. M. Lopez is with IMEC, Kapeldreef 75, 3001 Leuven, Belgium. 


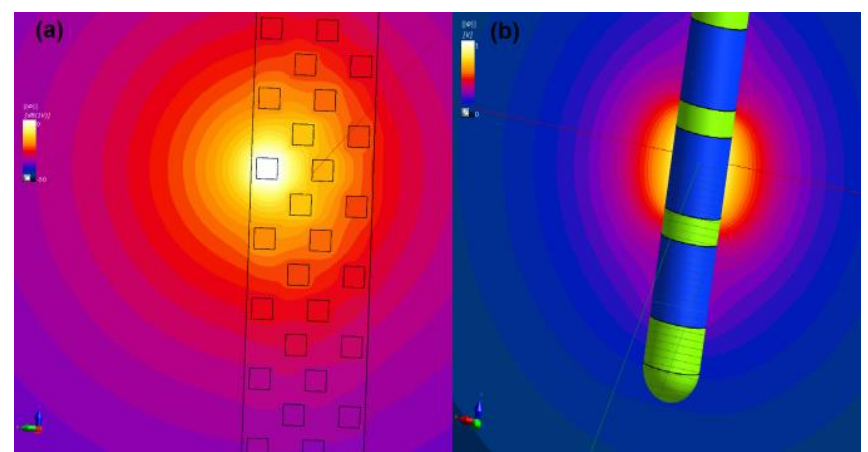

Figure 1. Potential distributions induced by application of $1 \mathrm{~V}$ to a single electrode. Bright colours indicate a higher electrical potential. The figures do not share the same scale. (a) High resolution recording probe (4.45 $\mu \mathrm{A}$ injection). (b) Conventional lead for deep brain stimulation (Medtronic 3889) (0.846 mA injection).

Here, $\phi$ is the electric potential and $\sigma$ is a uniform conductivity $\left(\sigma=0.1 \frac{\mathrm{s}}{\mathrm{m}}\right)$. First, simulations are performed with a single active electrode, by imposing $1 \mathrm{~V}$ on the electrode-patch and $0 \mathrm{~V}$ at the outer boundary of the simulation domain (Dirichlet conditions). Vanishing flux conditions are applied at the floating electrode patches and insulated parts of the DBS-leads (Neumann-conditions). Second, the injected current is calculated by integrating the current density in Sim4life over a surface, enclosing the active electrode. It was observed that the electrode impedance did only depend on the transversal electrode location (close or far from the lead's edge), and not on the longitudinal position. The potential distribution $\phi$ for the general case of current injection through multiple active electrodes is then determined through rescaling, translation and linear superposition of the potential distributions.

To determine the volume of tissue activated, the approach used in Martens et al. [15] is followed, i.e. the VTA is calculated by thresholding the activating function $A F$ to $20 \mathrm{mV}$. The activating function was first introduced by Rattay $[16,17,18]$, and is given by:

$$
A F_{z}(x, y, z)=\phi(x, y, z-\Delta z)-2 \phi(x, y, z)+\phi(x, y, \Delta z)
$$

Here, $\Delta z=0.5 \mathrm{~mm}$ is the distance between two nodes of Ranvier, under the assumption that intermodal myelin has infinite impedance. This is a typical intermodal distance, for basal ganglia fibres with a diameter of $5 \mu m[15,19]$. The neuronal fibres are considered to be aligned with the neuronal probe (i.e. oriented in the z-direction), cfr. [15]. $A F_{z}$ appears in the right-hand side of the neuronal cable equation, such that positive and negative $A F_{z}$ will favor depolarization and hyperpolarization, respectively. A good initial approximation of the VTA is obtained through thresholding the $A F_{z}$ to $20 \mathrm{mV}$, which is the activation threshold corresponding with a pulse duration $\tau_{p}$ of $60 \mu s$ [20].

Due to the small surface areas of the electrode patches on the high-resolution recording probe, it is important to determine the maximal charge that can safely be injected. The Shannon-equation describes the limit between damaging and non-damaging electrical stimulation [12] and is based on a data-set obtained by McCreery [21, 22]:

$$
I \tau_{p}=\sqrt{10^{k} A}
$$

Here, $A$ is the electrode surface area, expressed in $\mathrm{cm}^{2}$ and $Q=I \tau_{p}$ is the injected charge in $\mu C$. The adjustable parameter $k$ is typically chosen between 1.5 and 2. E.g., $k=$ 1.5 is used in the original publication by Shannon [12], while $k=1.75$ can be used to obtain the maximum injected charge density of $30 \mu \mathrm{C} / \mathrm{cm}^{2}$ for which the first deep brain stimulator (Medtronic Activa Tremor Control System) was approved in the US [23, 24]. This maximum charge density $D=$ $30 \mu \mathrm{C} / \mathrm{cm}^{2}$ corresponds to the electrode area $A=0.06 \mathrm{~cm}^{2}$ of the Medtronic-lead (corresponding to $I=30 \mathrm{~mA}$ for $\tau_{p}=$ $60 \mu s$ in this study).

Equation (2) is meant as a near-field safety limit, that is dependent on both the charge density and the injected charge. The Shannon-equation attributes this codependence of the safety limit on injected charge and charge density to the inhomogeneous distribution of the current density over the electrode patch [12]. Higher current densities are found at the electrode edges, resulting in a linear proportionality between the safe current and the edge length of the patch (note the square root in (2)). However, a modeling study performed by McIntyre and Grill demonstrates that the potential distribution induced by conical micro-electrodes with suface areas between $100 \mu \mathrm{m}^{2}$ and $1000 \mu \mathrm{m}^{2}$ can be approximated by a point source for distances larger than $50 \mu \mathrm{m}$ [25]. This result indicates that the near-field assumption in the Shannonequation is not valid for micro-electrodes. Instead, a safety limit on the injected charge $(Q<4 n C / p h)$ emerges from experimental data on micro-electrode stimulation [14, 26, 27].

Both the Shannon-equation for macro-electrodes and the $4 n C / p h$ limit should be used with care, because damaging levels will also depend on factors not explicitly taken into account, such as the stimulation duration, pulse duration, duty cycle, pulse rate, electrode shape and material [3, 12, 14]. Furthermore, also the type of neuronal tissue is important, due to differences in the packing density, fibre diameter and neuronal metabolism [3, 14]. However, in this explorative study, that aims to investigate the potential of a high-recording probe for micro-stimulation, we will use the mentioned limits as guidelines for the current that can be safely injected by the electrodes.

\section{RESULTS AND DISCUSSION}

Current injections well below the safety limits are able to induce a volume of activated tissue with a diameter of several millimeters, with the conventional DBS-lead (see Fig. 2). In Fig. 2, it is demonstrated how the VTA can be shifted along the conventional lead, by steering the current to different electrodes. The precision with which the VTA can be placed, is determined by the separation between the electrodes. In the case of the Medtronic 3389 lead, millimeter accuracy in the localization of the VTA can be achieved. In contrast, targeting of the VTA with the high-resolution probe can be done with significantly higher accuracy, as is shown in Fig. 3.

Computational results indicate that micrometer precision targeting of stimulation regions can be achieved with the existing high-resolution recording probe. In Fig. 3, it is shown that small current injections $(0.03 \mathrm{nC} / \mathrm{ph})$ can move the VTA 

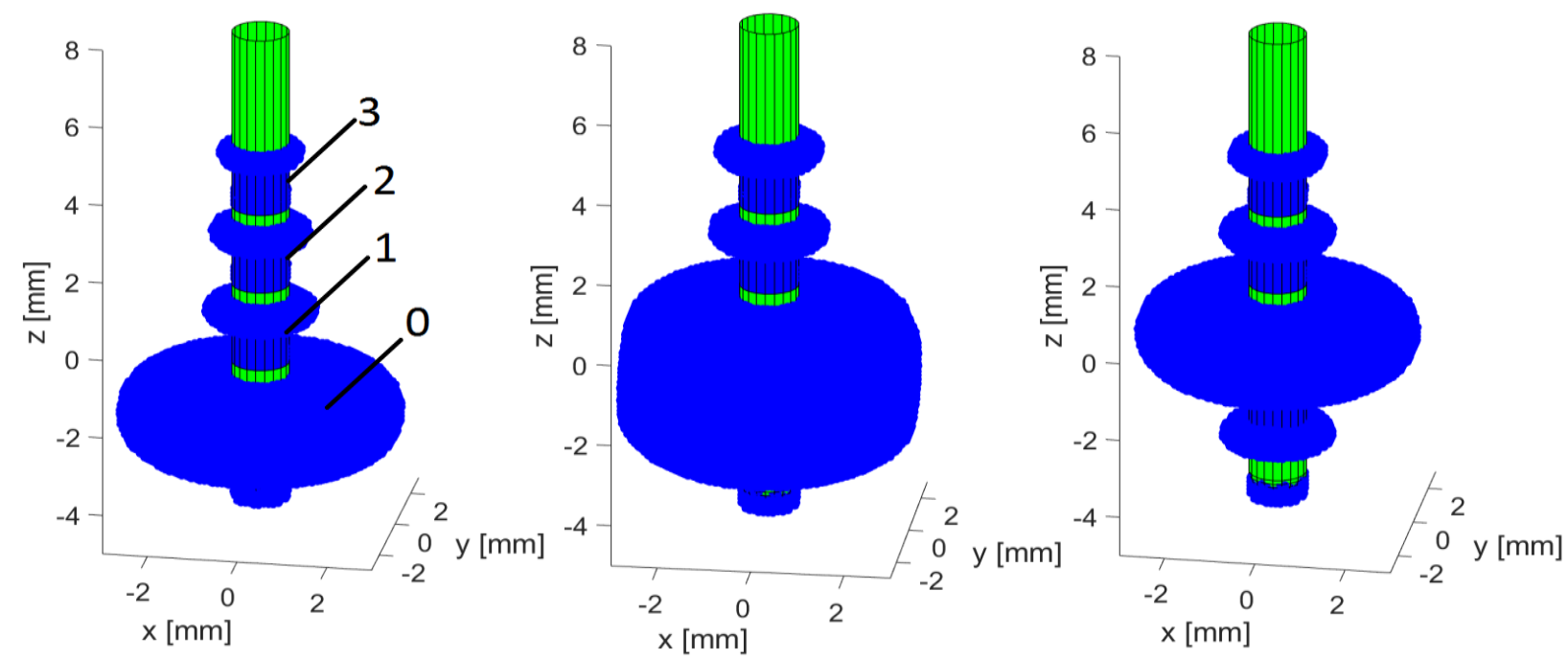

Figure 2. Volume of tissue activated by a conventional (Medtronic) DBS lead. Insulated parts are in green and electrodes are in blue. The blue volume is the region in which the activating function exceeds $20 \mathrm{mV}$ (VTA). (Left) $-2.5 \mathrm{~mA}$ injection by electrode 0 , (middle) $-1.25 \mathrm{~mA}$ injection by electrode 0 and electrode 1 , (right) $-2.5 \mathrm{~mA}$ injection by electrode 1 .

between the different active patches. Injecting current at the safety limit $(I=-66.6 \mu A$ or $4 n C / p h)$ through 150 subsequent electrode patches (corresponding to $1.5 \mathrm{~mm}$ in the $\mathrm{z}$-direction) results in neuronal activation $(A F \geq 20 \mathrm{mV})$ in a region of several millimeter (results not shown). This result is similar to the results in Fig. 2 (left or right), where $I=-2.5 \mathrm{~mA}$ is injected through a single electrode with an axial length of $1.5 \mathrm{~mm}$ on the Medtronic lead. However, in the case of the Medtronic 3389 lead, this volume of activated tissue is achieved with charge injection well below the safety limit of $30 \mu \mathrm{C} / \mathrm{cm}^{2}$. Consequentially, the application of the high-resolution probe would focus on the benefits of more local and precise neurostimulation, potentially accessing the topological organization of the subthalamic nucleus. Furthermore, the relatively small size of the probe would reduce the risk of surgical-related complications.

VTA-(442)(-0.5 $\mu \mathrm{A})$ stimulation

VTA-(442)(-0.25 $\mu \mathrm{A})(445)(-0.25 \mu \mathrm{A})$ stimulation

VTA-(445)(-0.5 $\mu \mathrm{A})$ stimulation
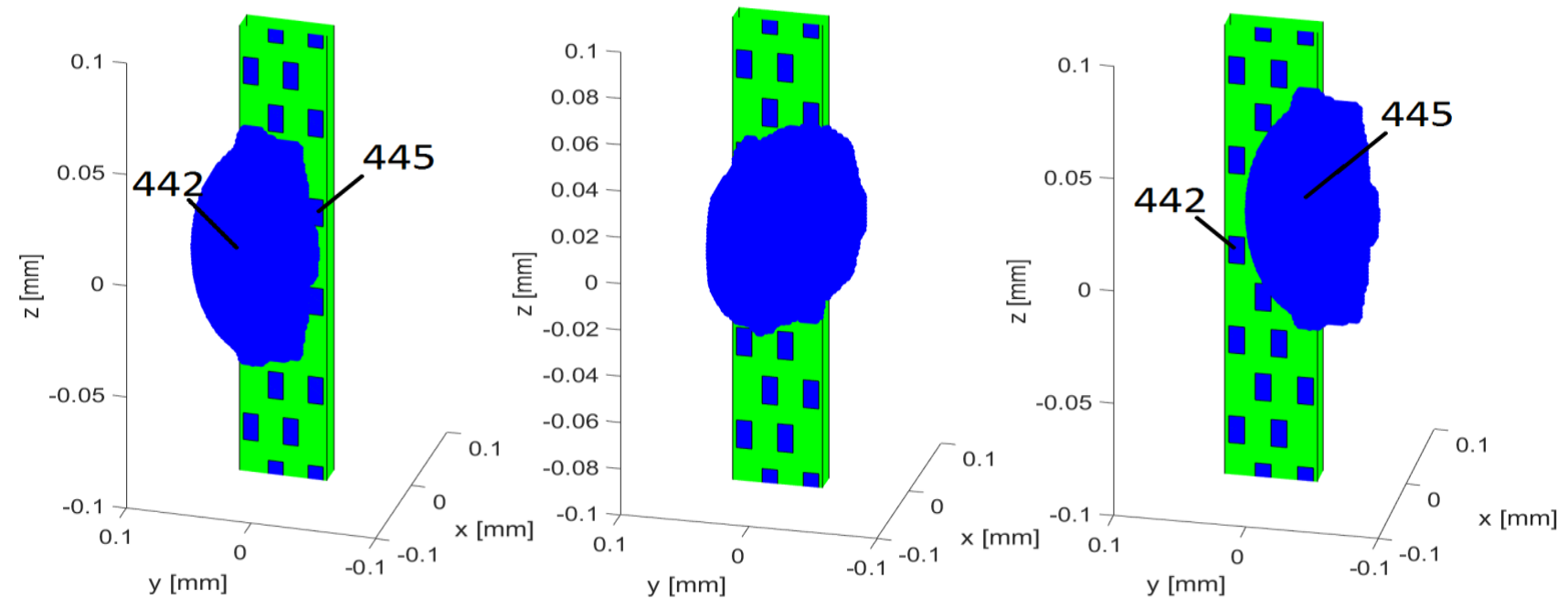

Figure 3. Volume of tissue activated by a high-resolution recording probe. Insulated parts are in green and electrode patches are in blue. The blue volume is the region in which the activating function exceeds $20 \mathrm{mV}$ (VTA). (Left) $-0.5 \mu A$ injection by electrode-patch 442 , (middle) $-0.25 \mu \mathrm{A}$ injection by electrode patch 442 and patch 445 , (right) $-0.5 \mu \mathrm{A}$ injection by electrode patch 445 . 


\section{CONCLUSIONS}

Computational modeling was used to investigate the stimulating capability of an existing high-resolution probe. The neurorecording probe is able to target the VTA with micrometer precision. Furthermore, a reasonable volume of activated neurons can be achieved by imposing $I=-66.6 \mu \mathrm{A}$ on 150 subsequent electrode patches. However, this current $I$ corresponds to a proposed safety limit for charge injection with micro-electrodes. It is not well understood, how simultaneous injection of charge through multiple neighbouring electrodes would impact the safe charge limit. Furthermore, other factors such as the pulse rate and duty cycle, could influence safety limits as well. Consequentially, application of the high-resolution probe would focus on the benefits of local and precise neurostimulation, instead of large volumes of activated tissue. Furthermore, the small size of the probe is likely to result in a reduction of surgical-related sideeffects, such as hemorrhage. The authors intend to use this work as a basis for future research, which will include more detailed computational simulations (neuronal membrane dynamics, tissue inhomogeneity and anisotropy, ...) and in vivo experiments to establish safety and efficacy of the probe for therapeutic applications. Furthermore, we will investigate several options to maximize the probe's performance and flexibility, such as increasing the electrode size (i.e. to use the whole shank width), investigating new electrode materials and shapes, influence of stimulation patterns, etc.

\section{REFERENCES}

[1] A. L. Benabid, "Deep brain stimulation for Parkinson's disease," Current opinion in neurobiology, vol. 13, no. 6, pp. 696-706, 2003.

[2] A. L. Benabid, P. Pollak, D. Gao, D. Hoffman and P. Limousin et al., "Chronic electrical stimulation of the ventralis intermedius nucleus of the thalamus as a treatment of movement disorders," Journal of neurosurgery, vol. 84, no. 2, pp. 203-214, 1996.

[3] W. M. Grill, "Safety considerations for deep brain stimulation: review and analysis," Expert review of medical devices, vol. 2, no. 4, pp. 409-420, 2005.

[4] S. Afsharpour, "Topographical projections of the cerebral cortex to the subthalamic nucleus," J. Comp. Neurol., vol. 236, pp. 14-28, 1985.

[5] A. Abosch, W. D. Hutchinson, J. A. Saint-Cyr, J. O. Dostrovsky and A. M. Lozano, "Movement-related neurons of the subthalamic nucleus in patients with Parkinson disease," J. Neurosurg., vol. 97, pp. 1167-1172, 2002.

[6] R. M. Beckstead, "A reciprocal axonal connection between the subthalamic nucleus and the neurostriatum in the cat," Brain Res., vol. 275, pp. 137-142, 1983.

[7] P. Romanelli , G. Heit, B. C. Hill, A. Kraus, T. Hastie and H. M. Bronte-Stewart, "Microelectrode recording revealing a somatotopic body in the subthalamic nucleus in humans with Parkinson disease," J. Neurosurg, vol. 100, pp. 611-618, 2004.

[8] F. A. Mettler and G. M. Stern, "Somatotopic localization in the rhesus subthalamic nucleus," Arch. Neurol., vol. 7, pp. 328-329, 1962.

[9] D. McCreery, A. Lossinsky, V. Pikov and X. Liu, "Microelectrode array for chronic deep-brain microstimulation and recording," IEEE Trans. on Biomed. Eng., vol. 53, no. 4, pp. 726-737, 2006.
[10] A. Priori, G. Foffani, L. Rossie and S. Marceglia, "Adaptive deep brain stimulation (aDBS) controlled by local field potential oscillations," Experimental neurology, vol. 245, pp. 77-86, 2013.

[11] J. J. Jun, N. A. Steinmetz, J. H. Siegle, D. J. Denman and M. Bauza et al., "Fully integrated silicon probes for high-density recording of neural activity," Nature, vol. 551, pp. 232-236, 2017.

[12] R. V. Shannon, "A model of safe levels for electrical stimulation," IEEE Transactions on Biomedical Engineering, vol. 39, no. 4, pp. 424-426, 1992.

[13] D. R. Merill , M. Bikson and J. G. Jefferys, "Electrical stimulation of excitable tissue: design of efficacious and safe protocols," Journal of neuroscience methods, vol. 141, no. 2, pp. 171-198, 2005.

[14] S. F. Cogan, K. A. Ludwig, C. G. Welle and P. Takmanov, "Tissue damage thresholds during therapeutic electrical stimulation," Journal of neural engineering, vol. 13, no. 2, 2016.

[15] H. C. F. Martens, E. Toader, M. J. Decré, D. J. Anderson, R. Vetter and D. R. Kipke et al., "Spatial steering of deep brain stimulation volumes using a novel lead design," Clinical neurophysiology, vol. 122, no. 3, pp. 558-566, 2011.

[16] F. Rattay and M. Aberham, "Modeling axon membranes for functional electrical stimulation," IEEE Transactions on Biomedical Engineering, vol. 40, no. 12, pp. 1201-9, 1993.

[17] F. Rattay, "Analysis of models for external stimulations of axons," IEEE Trans. Biomed. Eng., Vols. BME-33, pp. 974-977, 1986.

[18] F. Rattay, "Electrical nerve stimulation," New York: Springer, 1990.

[19] W. J. C. Verhaart, "Fiber analysis of the basal ganglia," J. Comp. Neurol., vol. 933, pp. 425-40, 1950.

[20] C. C. McIntyre, S. Mori, D. L. Sherman, N. V. Thakor and J. L. Vitek, "Electric field and stimulating influence generated by deep brain stimulation of the subthalamic nucleus," Clin. Neurophysiol., vol. 115, pp. 589-95, 2004.

[21] D. B. McCreery, W. F. Agnew, T. G. H. Yuen and L. Bullara, "Comparison of neural damage induced by electrical stimulation with faradaic and capacitor electrodes," Ann. Biomed. Eng., vol. 16, pp. 463-481, 1968.

[22] D. B. McCreery, W. F. Agnew, T. G. Yuen and L. Bullara, "Charge density and charge per phase as cofactors in neural injury induced by electrical stimulation," IEEE Trans. Biomed. Eng., vol. 37, pp. 9961001, 1990.

[23] U. FDA, "Medtronic Activa Tremor Control System," Summary of Safety and Effectiveness, 1997.

[24] A. M. Kuncel and W. M. Grill, "Selection of stimulus parameters for deep brain stimulation," Cin. Neurophysiol., vol. 115, pp. 2431-41, 2004.

[25] W. M. McIntyre and W. M. Grill, "Finite element analysis of the current density and electric field generated by metal microelectrodes," Ann. Biomed Eng., vol. 29, pp. 227-35, 2001.

[26] D. B. McCreery, T. G. H. Yuen, W. F. Agnew and L. A. Bullara, "Stimulus parameters affecting tissue injury during microstimulation in the cochlear nucleus of the cat," Hear. Res., vol. 77, pp. 105-15, 1994.

[27] D. McCreery, V. Pikov and P. R. Troyk, "Neuronal loss due to prolonged controlled-current stimulation with chronically implanted microelectrodes in the cat cerebral cortex," J. Neural Eng., vol. 7, 2010 . 